\title{
Complement monitoring of carbon nanotubes
}

To the Editor - In their survey describing the promises and challenges of carbon nanotubes in imaging and therapeutics, Kostarelos and colleagues ${ }^{1}$ state that “...chemically functionalized [carbon] nanotubes have been shown by many groups to be more biocompatible (no immune or acute inflammatory responses) than pristine nanotubes." We point out below in vitro and in vivo studies that show surface-modified carbon nanotubes can trigger immune responses, notably activation of the complement system ${ }^{2-4}$.

The complement system is a network of over thirty different proteins that orchestrate innate and acquired immunity. Microbial invaders and nanoparticulate systems, depending on their surface properties, size and shape, may trigger the complement cascade through any of the three established classical, alternative and lectin pathways, and more than one pathway may be involved in many cases. Activation of the complement system primes the surface of the invaders with complement proteins for rapid recognition and ingestion by phagocytic cells.

Complement activation further generates anaphylatoxins and chemoattractants, and these may potentially trigger the release of a variety of secondary mediators that could, in turn, initiate pseudoallergic responses in sensitive individuals ${ }^{5}$. These responses are often associated with cardiopulmonary disturbance and other related symptoms such as skin reactions, and have been noted in some patients receiving regulatory-approved nanomedicines, such as liposomes and micelles, and contrast agents $^{5-7}$. The incidence of pseudoallergic responses was recently estimated to be as high as $77 \%$ of all immune-mediated hypersensitivity reactions. Therefore, understanding the molecular basis of complement activation is relevant to the design of safer nanomedicines and carbon nanotubes.
Sim and colleagues ${ }^{2}$ demonstrated in vitro that pristine single-walled carbon nanotubes trigger the complement system by the classical pathway, whereas doublewalled carbon nanotubes are capable of doing this by both the classical and alternative pathways. Carbon nanotubes that are covalently functionalized with either $\varepsilon$-caprolactam or L-alanine were further shown to activate the complement system but to a lesser extent than pristine nanotubes, and it was suggested that chemical functionalization may afford some degree of protection against complement activation $^{3}$. However, it is important to note that from the immunological point of view even a small amount of complement activation may lead to adverse reactions in over-sensitive individuals.

For many proposed biomedical applications, carbon nanotubes require prolonged circulation times in the blood, and this is achieved by coating the surface with certain polymeric materials such as poly(ethylene glycol) (PEG) and poloxamers ${ }^{5,8}$. PEG-phospholipid conjugates in both monomeric and micellar forms do not activate the complement system ${ }^{7}$. Yet, in our studies ${ }^{4}$, we found that singlewalled carbon nanotubes non-covalently coated with amino- and methoxy- $\mathrm{PEG}_{5000^{-}}$ phospholipid (average length $250 \mathrm{~nm}$ and 1-5 $\mathrm{nm}$ wide) activated the complement cascade in human sera through the lectin pathway, and induced complement activation in vivo.

In our opinion, current surfacemodification methodologies (covalent or non-covalent) do not necessarily prevent the activation of the complement system, but rather switch activation from one pathway to another. We believe that the inherent property of the carbon-nanotube surface plays a role in modulating complement activation because current surface-modification strategies do not uniformly cover the nanotube surface.
Understanding the molecular basis of complement events, however, may provide a viable platform for designing safer carbon nanotubes with possible applications in medicine. Nevertheless, the chances for preventing complement-activation-related pseudoallergy might be improved using complement inhibitors ${ }^{6}$.

Single-walled carbon nanotubes that have been covalently functionalized with a neutralizing viral coat protein have been shown in vivo ${ }^{9}$ to generate high levels of protective and virus-neutralizing antibodies. Although this means nanotubes could potentially deliver candidate vaccine antigens to peripheral dendritic cells and therefore act as particulate adjuvants, the adjuvanticity of the peptide-functionalized carbon nanotubes is consistent with their ability to activate the complement system ${ }^{2}$. In conclusion, the interaction of nanomaterials (including carbon nanotubes) with the immune system deserves special attention.

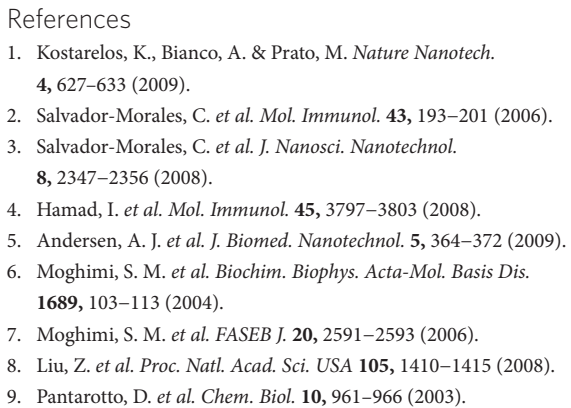

\section{S. M. Moghimi ${ }^{1 \star}$ and A. C. Hunter ${ }^{2}$}

${ }^{1}$ Centre for Pharmaceutical Nanotechnology and Nanotoxicology, Department of Pharmaceutics and Analytical Chemistry, University of Copenhagen, Universitetsparken 2, DK2100 Copenhagen Ø, Denmark, ${ }^{2}$ Molecular Targeting and Polymer Toxicology Group, School of Pharmacy, University of Brighton, Brighton BN2 4GJ, UK.

*e-mail: momo@farma.ku.dk
Authors' reply - In our Progress Article ${ }^{1}$, we defined 'pristine' carbon nanotubes as unmodified; 'coated' as surface modified by non-covalent coating with lipid, polymers or surfactants; and 'functionalized' as chemically modified by either acid treatment or other covalent conjugation reactions. With this clarification in mind, the statement by Moghimi and Hunter points out the considerable differences in complement activation reported for pristine and coated carbon nanotubes ${ }^{2,3}$ compared with chemically functionalized tubes ${ }^{4}$. Based on published in vitro studies ${ }^{4}$, chemically functionalized nanotubes have been shown to considerably reduce the level of complement activation compared with pristine or coated nanotubes. We also 